\title{
Quantitative Analysis of Skin Erythema Due to Laser Hair Removal: A Diffusion Optical Spectroscopy Analysis
}

\author{
Mozhdeh Babadi', Ezeddin Mohajerani ${ }^{1 *}$, Leila Ataie-Fashtami ${ }^{2}$, Nasrin Zand ${ }^{3}$, Afshan Shirkavand ${ }^{1,3}$ \\ ${ }^{1}$ Laser and Plasma Research Institute, Shahid Beheshti University, Tehran, Iran \\ ${ }^{2}$ Department of Regenerative Medicine, Royan institute For Stem Cell Biology and Technology, ACECR, Tehran, Iran \\ ${ }^{3}$ Department of Medical Laser, Medical Laser Research Center, ACECR, Tehran, Iran
}

\author{
*Correspondence to \\ Ezeddin Mohajerani, Professor \\ of Physics, Laser and Plasma \\ Research Institute, Shahid \\ Beheshti University, Tehran, \\ Iran \\ Tel: +982129902040 \\ Email: e-mohajerani@sbu.ac.ir
}

Published online February 25, 2019

\begin{abstract}
Introduction: Laser hair removal needs an accurate understanding of tissue structure and chromophores content in order to optimize the selection of laser irradiation parameters. None of the optimized laser therapy might lead to side effects in skin tissue such as severe erythema, burn, scar etc. Therefore, guidance by a noninvasive real-time diagnostic method like optical spectroscopy technique is beneficial. The purpose of this survey is to analysis the skin hemoglobin spectrum quantitatively before and after hair removal laser irradiation to minimize the side effects of the procedure.

Methods: To carry out a spectroscopy study, a halogen-tungsten light source was used in the wavelength region of 400-700 nm on an ocean optic device. The measurements were made on the facial area under identical conditions. Total 19 volunteers for laser hair removal by gentle laser Candela, ranging 14- 49 years old, were included in the study. A total of 18 spectra were taken from each person, 9 spectra before hair removal as a reference and 9 subsequent spectra. Colorimetry was done for all acquired before and after spectrums using Origin software (version 8.6). Then, the erythema index derived for each spectrum. Statistical analysis of correlation and normalization in colorimetry data were done using data analysis by SPSS (version 16).

Results: Spectra analysis, before and after optical reflectance spectrums in laser hair removal procedure, revealed the subpeak derivation, and concentration on special visible wavelength 510$610 \mathrm{~nm}$. We studied the changes of skin chromophores absorption. The derived erythema index [E] and colorimetry parameters $a^{*}, b^{*}, I^{*}$ were compared and correlated statistically. There was a statistically considerable direct linear correlation between $\mathrm{a}^{*}$ and $\mathrm{E}$ while inverse linear correlation was observed for $\mathrm{I}^{*}$ and $\mathrm{E}$ and no correlation for $\mathrm{b}^{*}$ and $\mathrm{E}$.

Conclusion: Diffuse reflectance spectroscopy showed its potency as an accurate, noninvasive realtime as complementary method for laser treatment to detect erythema as a complication of the method, in order to optimize the parameters based on the tissue characteristics in various candidates. Keywords: Laser hair removal; Erythema; Diffuse reflectance; Spectrum analysis; Colorimetry.
\end{abstract}

\section{Introduction}

The skin as the external organ of the human body consists of three main layers epidermis, dermis, and hypodermis. ${ }^{1}$ Visual subjective evaluation of the skin disease diagnosis and its response to treatment has long been of interest to physicians. However, this method leads less information on complication results. The use of more modern analytical technologies can be very effective as a complementary objective method in diagnosis and treatment. The biggest problem in medical science is the study of skin and the examination of skin diseases in a noninvasive, precise manner with minimal injuries. Accordingly, optical techniques have been widely used in recent years. Reflectance diffusion spectroscopy is one of these optical techniques that enable low-cost and non- destructive provision of information on morphology, optical and biochemical properties of the skin tissue. ${ }^{2}$

In medicine, the laser was used as a hair removal tool in 1996 for the first time. Failure to choose optimal laser irradiation parameters such as energy density, pulse duration, etc, and variety of optical properties of the different skin types lead to complications such as burn, erythema, changes of skin pigment changes and scar following the laser irradiation, which in turn results in patient dissatisfaction. ${ }^{3,4}$ Erythema (redness), as a side effect due to laser therapy, leads increased blood flow or hyperemia of blood capillaries in the subcutaneous layers, which in turn leads to the appearance of red spots on the skin surface. This complication is caused by various causes such as injury, heat, insects, drug sensitivity, and 
epilation or laser hair removal, and all factors leading to the dilation of the capillaries and ultimately redness.,

Since the physician uses subjective assessment to evaluate the skin condition after laser hair removal in most cases, and standard objective assessment methods such as biopsy and histopathologic tests are time-consuming and invasive, there is a vital need for a complementary method with minimal injury to the tissue structure. Optical spectroscopy is suggested in this case. ${ }^{7,8}$ Diffusive reflection-based spectrometry technique, as well as its application in colorimetry is widely used as a simple, fast, noninvasive and precise method for the investigation of biological tissues. Approximately $94 \%$ of the light reflected the surface of the tissue penetrates into it and undergoes multi-absorption and scattering events, and part of this penetrated light comes back to the skin surface, which is called a diffusive reflection. This technique is able to provide information on the morphology and biochemical properties of the tissue. ${ }^{9}$ The spectrometry is based on the interaction of the light beam with the biological tissue. The dominant phenomena occurring during light-tissue interaction include reflection, refraction, scattering, and absorption. Each of these phenomena provides very useful and effective information on the biological tissue. Scattering occurs due to the presence of micrometric components such as collagens, microorganisms, and lipids. ${ }^{10}$ The fact that which phenomenon is most dominant depends on the tissue undergoing irradiation and the wavelength of the incoming beam. In this regard, the wavelength is a very important factor, on which the coefficient of refraction, absorption, and scattering depends. ${ }^{11}$ The CIE-Lab model-based colorimetry technique (due to its proximity to the human eye) provides a quantitative review of the information received from the skin by the three parameters of $1^{*}, a^{*}$ and $b^{*}$. $1^{*}$ indicates the intensity of light ranging from 0 [black] to 100 [white]. a* indicates the sample color on a scale ranging from green (negative values) to red (positive values) and $b^{*}$ denotes the sample color on a scale ranging from blue (negative values) to yellow (positive values). ${ }^{12,13}$ Considering that absorption mainly occurs in the skin tissue by water molecules and macromolecules, such as proteins and chromophores, including hemoglobin, melanin, beta-carotene and bilirubin pigments, our main focus is on the hemoglobin pigment in order to determine the effect of the laser on it and high absorption in the visible region. ${ }^{14-16}$ The characteristic of blood absorption in the visible region of the spectrum is mainly affected by the hemoglobin. Hemoglobin, an iron-containing protein, gives blood its red color. In addition, hemoglobin plays an important role in keeping the shape of red blood cells. There are two types of hemoglobin in the blood: oxyhemoglobin and deoxyhemoglobin. Oxyhemoglobin absorbs visible light in the wavelengths of 420, 542 and $577 \mathrm{~nm}$, and deoxyhemoglobin in the wavelengths of 430, 555 , and $760 \mathrm{~nm}$. In general, hemoglobin, like melanin, is more absorbed at shorter wavelengths. ${ }^{17,18}$

The aim of this research is to investigate the spectral behavior of skin hemoglobin pigment after laser irradiation and to use of CIE-Lab colorimetric method after erythema in patients undergoing laser hair removal in order to quantify their pigment data. Accordingly, after laser irradiation and the occurrence of erythema, the absorption spectrum of patients, as well as their subpeaks was studied in the visible range of the electromagnetic spectrum. The most effective spectral region is extracted to evaluate the changes in the hemoglobin pigment after irradiation as well as the amount of erythema. The erythema index is calculated based on the obtained data and its results are compared with the quantitative one from the colorimetry technique.

\section{Methods}

Patients

This research was conducted between Septembers to December 2016, in a medical laser clinic in Tehran under the supervision of dermatologists. During this time interval, totally 19 female volunteers, aged 14-49 years, were included for this study. First, the experiment process was explained to individuals, and participants were enrolled in the study with the full consent regarding research methodology. All considerations of laser hair removal procedure were applied. Moreover, the spectroscopy was done for all included patients no more than 10 minutes after laser hair removal.

\section{Hair Removal Laser System}

Alexandrite's laser with a wavelength of $755 \mathrm{~nm}$ was used in this research. Variable parameters given to the device by the physician are different depending on the density and thickness of the hair and the color of the skin and hair. The pulse energy density and pulse duration were chosen as two important laser irradiation parameters, according to the physician's diagnosis.

\section{Diffuse Reflectance Spectroscopy}

To carry out a spectrometric study, a halogen-tungsten light source was used in the wavelength region of 400-700 $\mathrm{nm}$. The source light is irradiated to the skin surface by the optical fiber. Part of the penetrated light is scattered and dispersed due to the internal structure of the skin and returns to the incident ray and leaves the skin through its surface. As shown in Figure 1, the light underwent the diffuse reflection process is then collected by a fiber optic based on a two-way probe. The reflection probe consists of seven fibers that lie adjacent to each other. Six fibers send light on the skin and the central fiber collects light scattered from the skin surface and transmits the information to the spectrometer (USB2000, Ocean Optics Inc).

After ensuring the calibration of the device, the measurements were made on their facial area under 


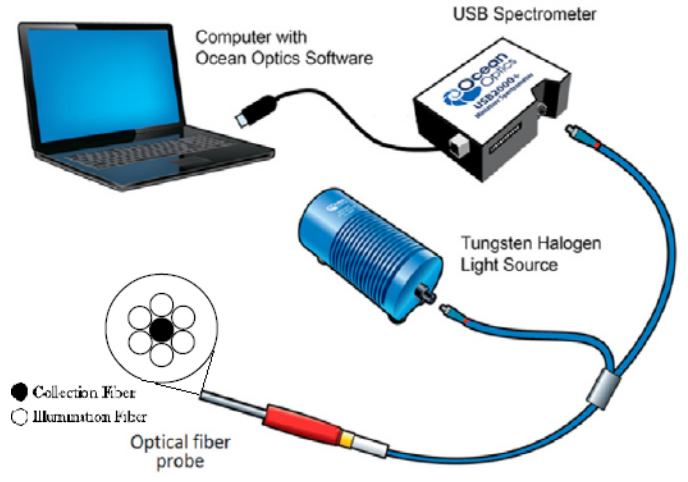

Figure 1. Set-up of Diffuse Reflectance Spectroscopy.

identical clinical conditions. A total of 18 is transmission spectra were taken from each patient, 9 spectra before hair removal as a reference and 9 subsequent spectra.

Information is transmitted through a spectrometer to a computer for quantitative analysis and then processed as a function of the wavelength by Origin version 8.6 software. According to equation 1, the absorption spectrum can be calculated from the inverse of the reflection logarithm at each wavelength using this software.

$\mathrm{A}(\lambda)=-\log R[\lambda]$

In equation $1, A$ demonstrates the absorption rate in $\lambda$ wavelength and $R$ is the reflection coefficient in that wavelength. Since absorption is obtained indirectly from the reflection spectrum, it is indicative of apparent absorption.

Considering the complex structure of the skin and the effect of melanin pigment as a strong absorbent, which affects the entire region of the visible spectrum, the corresponding subpeak Lorenz curves of equation 2 were plotted according to the absorption peaks of the hemoglobin and the bilirubin pigments in order to the investigate the effect of eliminating these unwanted agents on any of the absorption spectra. The present article also used the compatibility feature of Lorentzian functions found in the Origin software.

$y=y_{0}+\frac{2 A}{\pi} \frac{w}{4\left(x-x_{c}\right)^{2}+w^{2}}$

$y_{0}$ denotes the minimum spectrum value along the vertical axis, $A$ shows the area under the curve and $W$ is the maximum width setting. $x_{c}$ also shows the value of the center of the Lorentzian function subpeaks. Using the resulting subpeaks, it is possible to accurately evaluate each of the hemoglobin and bilirubin pigments independent of the other components present in the skin. The concept of hemoglobin with an erythema index (E) is estimated, which is proportional to the absorption intensity in this spectral range. The erythema index is later calculated by equation $3^{17}$ :

$$
\mathrm{E}=100\left[\mathrm{~A}_{560}+1.5\left[\mathrm{~A}_{575}+\mathrm{A}_{545}\right]-2\left[\mathrm{~A}_{510}+\mathrm{A}_{610}\right]\right]
$$

That $A$ is the apparent absorption intensity according to equation 1 for each wavelength.

\section{Colorimetry}

The spectroscopy software enables colorimetry capability based on the CIE-Lab model to examine skin erythema. Based on this capability, according to the skin reflection spectrum and the specification of the reference light $\left(D_{65}\right)$ and the type of observer (2 degrees), it is possible to obtain quantitative data $1^{*}, a^{*}$ and $b^{*}$ which, respectively, represent the brightness, the level of laser irradiationinduced erythema (redness) and the amount of jaundice (bilirubin pigment) present in the skin.

\section{Analysis}

All data related to the recorded spectra of patients were analyzed using the graphing software OriginPro 8.6. Origin graphs and analysis results can automatically update on data or parameter change, allowing you to create templates for repetitive tasks or to perform batch operations from the user interface, without the need for programming. OriginPro offers advanced analysis tools and apps for peak fitting, surface fitting, statistics and signal processing. In fact, this analytical software comes up with our requirements in statistical data analysis and graphing. Furthermore, statistical analysis of correlation and normalization in colorimetry data, we take advantage of the statistical advisor, using data analysis by SPSS (version 16).

\section{Results}

We documented for all patients the process of facial laser hair removal, using standard clinical photography. Figure 2 shows a typical before and after photo.

Acquired spectrums before hair removal as a reference and subsequent spectra after laser hair removal were recorded and saved for more analysis. Each of these spectra was averaged in the pre and post laser irradiation phases, area under each curve was calculated and the results were finally compared with each other (Figure $3 \mathrm{~A}$ ). The spectrum obtained from all specimens was $\mathrm{W}$ shape and similar due to the same structure of skin pigments, and the only difference was related to the intensity and absorption of light by these pigments from one person to another. In order to eliminate the complexity of the skin structure according to equation 2, each absorption
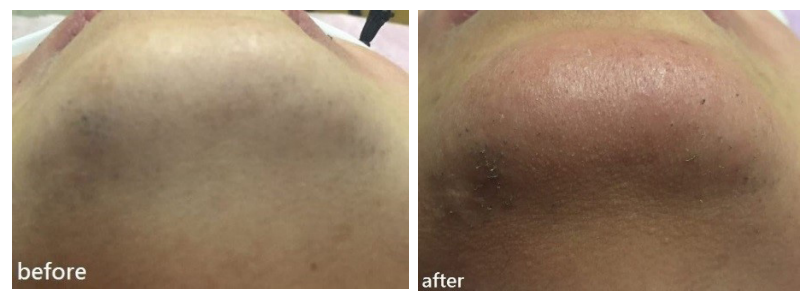

Figure 2. Facial Chin View Before and After Laser Irradiation. 

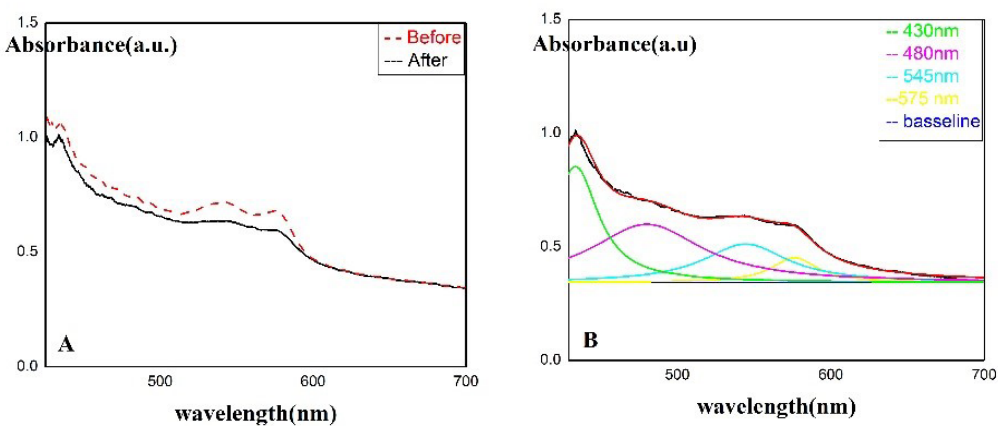

Figure 3. (A) Absorbance Spectra Before/After Laser Hair Removal (B) Derivation of Subcomponent Peaks $\left[R^{2}=0.99\right]$.

spectrum was decomposed into hemoglobin subpeaks (Figure 3B) at 430 [green diagram], 545 (bright-blue diagram) and $575 \mathrm{~nm}$ (yellow diagram) and bilirubin $480 \mathrm{~nm}$ (purple diagram) with a correlation coefficient of 0.99 , and the difference in subpeaks height were calculated and compared (Figure 3).

According to the results of the subpeaks investigation, the erythema index was calculated for each individual after laser post-irradiation, and the results were evaluated by the values obtained from the colorimetric test using a spectrometer and spectrum obtained from the skin.

As shown in Figure 3A, the apparent absorption spectrum of individuals was measured before and after laser irradiation and an increase is observed in the initial region of the visible spectrum as the wavelength range of 430-610 nm, after the laser irradiation (red spectrum). The results of matching the absorption intensity of the 2 diagrams at the end of the visible spectrum at the wavelength range of 610-700 $\mathrm{nm}$ show the lack of effect of laser irradiation on this region. Considering to the widespread absorption intensity of melanin pigment in the entire visible spectral region and the lack of effect of irradiation on the end of the spectrum, as well as the presence of hemoglobin and bilirubin pigment at the beginning of the spectral region and increasing the absorption intensity in this region, the area under the curve analysis is focused on the initial parts of the visible spectrum and these 2 pigments, which the percentage of change in the area under the curve is calculated before and after irradiation as shown in Table 1.

Since the study focuses on the initial part of the spectrum, this the initial part has the maximum absorption of hemoglobin and bilirubin pigments; therefore, to study the behavior of each of these two pigments after laser irradiation and also to eliminate the effect of melanin pigment absorption intensity, the subpeaks with a

Table 1. Intensity Differences of Skin and Hemoglobin- Bilirubin Chromophores Absorbance Spectra in Visible Wavelength, Before and After Irradiation

\begin{tabular}{|c|c|c|c|c|c|c|}
\hline \multirow{2}{*}{ Person } & \multirow{2}{*}{$\begin{array}{l}\text { Skin Type } \\
\text { [Diagnosis] }\end{array}$} & \multirow{2}{*}{$\begin{array}{l}\text { Difference in Absorption } \\
\text { [spectrum of skin] \% }\end{array}$} & \multicolumn{4}{|c|}{ Difference in Subpeaks } \\
\hline & & & $430 \mathrm{~nm}$ & $480 \mathrm{~nm}$ & $545 \mathrm{~nm}$ & $575 \mathrm{~nm}$ \\
\hline a & 2 & 0 & -0.014 & -0.013 & 0.004 & 0 \\
\hline$b$ & 2 & 7.02 & 0.126 & -0.005 & 0.098 & 0.087 \\
\hline c & 2 & 0 & -0.013 & 0.047 & 0.046 & 0.051 \\
\hline$d$ & 2 & 5.99 & -0.033 & 0.01 & 0.032 & 0.07 \\
\hline e & 2 & 4.21 & 0.118 & -0.024 & 0.101 & 0.038 \\
\hline$f$ & 2 & 21.9 & 0.216 & 0.073 & 0.146 & 0.159 \\
\hline g & 3 & 1.52 & -0.016 & 0.036 & 0.037 & 0.061 \\
\hline $\mathrm{h}$ & 3 & 6.1 & 0.085 & 0.001 & 0.089 & 0.074 \\
\hline $\mathrm{i}$ & 3 & 5.57 & 0.135 & 0.007 & 0.096 & 0.036 \\
\hline j & 3 & 22.52 & 0.191 & 0.079 & 0.168 & 0.187 \\
\hline k & 3 & 8.3 & 0.056 & 0.029 & 0.078 & 0.096 \\
\hline I & 3 & 2.03 & 0.013 & 0.007 & 0.011 & 0.035 \\
\hline $\mathrm{m}$ & 3 & 11.67 & 0.637 & 0.151 & 0.123 & 0.172 \\
\hline $\mathrm{n}$ & 3 & 12.68 & 0.266 & 0.048 & 0.11 & 0.116 \\
\hline o & 4 & 4.17 & 0.055 & 0.009 & 0.056 & 0.081 \\
\hline$p$ & 4 & 3.75 & -0.023 & 0.011 & 0.055 & 0.059 \\
\hline$q$ & 4 & 3.9 & 0.005 & 0.04 & 0.08 & 0.072 \\
\hline$r$ & 4 & 3.75 & 0.025 & -0.038 & 0.111 & 0.058 \\
\hline s & 4 & 2.43 & -0.02 & 0.031 & 0.051 & 0.097 \\
\hline
\end{tabular}


correlation coefficient of 0.99 are considered according to equation 2. Figure 3B shows the Lorentzian distribution of the absorption spectrum of each chromophore occurred separately and independently of other factors. The difference in height of absorbance intensity for these two pigments [in each wavelength separately] was expressed before and after the laser irradiation in Table 1.

Investigating the subpeaks is a better way to describe the process of changes, the cause, and the response mechanism of hemoglobin and bilirubin chromophores after laser irradiation. Table 1 shows the irregular process of change in 430 [hemoglobin] and $480 \mathrm{~nm}$ [bilirubin] and lack of dependence on bilirubin pigment. Also, from the point of view of skin tissue morphology, the study of the post-irradiation apparent absorption spectrum of the skin at the range of $510-610 \mathrm{~nm}$ is due to the absorption of hemoglobin and blood. The hemoglobin concept is estimated in accordance with equation 3 using the erythema index $[\mathrm{E}]$ and compared with the colorimetric parameters of $1^{*}, a^{*}$ and $b^{*}$. Figure 4 shows the behavior of the skin's erythema index after laser irradiation along with three colorimetric parameters based on computational and experimental data. Erythema is usually estimated by $a^{*}$ parameter and skin color by $b^{*}$ and $l^{*}$ parameters. The positive values of $\mathrm{a}^{*}$ and $\mathrm{b}^{*}$ were also taken into account.

It would possible to express the dispersion or dependence of this index on $\mathrm{l}^{*}, \mathrm{a}^{*}$ and $\mathrm{b}^{*}$ parameters by comparing the erythema index obtained from the absorption spectrum with the information on these parameters. Figure $4 \mathrm{~A}$ shows a linear relationship between the erythema index and the redness parameter $\mathrm{a}^{*}$ with the linear gradient with a correlation coefficient $\mathrm{R}^{2}=0.82$ in accordance with equation 4 .

\section{E index $=4.245 a^{*}+3.025$}

In this linear relationship, the gradient acquired 4.245 between this parameter and the erythema index, which indicates that the results of this test are consistent with the values calculated for the erythema index.

In regards, the statistical analysis, the normality of $\mathrm{a}^{*}$ and E factors was confirmed using one-sample KolmogorovSmirnov test while there was a statistically considerable direct linear correlation between them (Table 2).

Moreover, the analysis was repeated for the normality of $2 \mathrm{~b}^{*}$ and $\mathrm{E}$ factors were confirmed using one-sample Kolmogorov-Smirnov test and more analysis showed no correlation using Pearson correlation (Table 3).

The normality of two $\mathrm{l}^{\star}$ and $\mathrm{E}$ factors was confirmed using one-sample Kolmogorov-Smirnov test and more analysis showed inverse considerable correlation using Pearson correlation (Table 4).

\section{Discussion}

The aim of the present study was to investigate the effect of laser hair removal on the behavior of skin chromophores and the intensity of the skin erythema
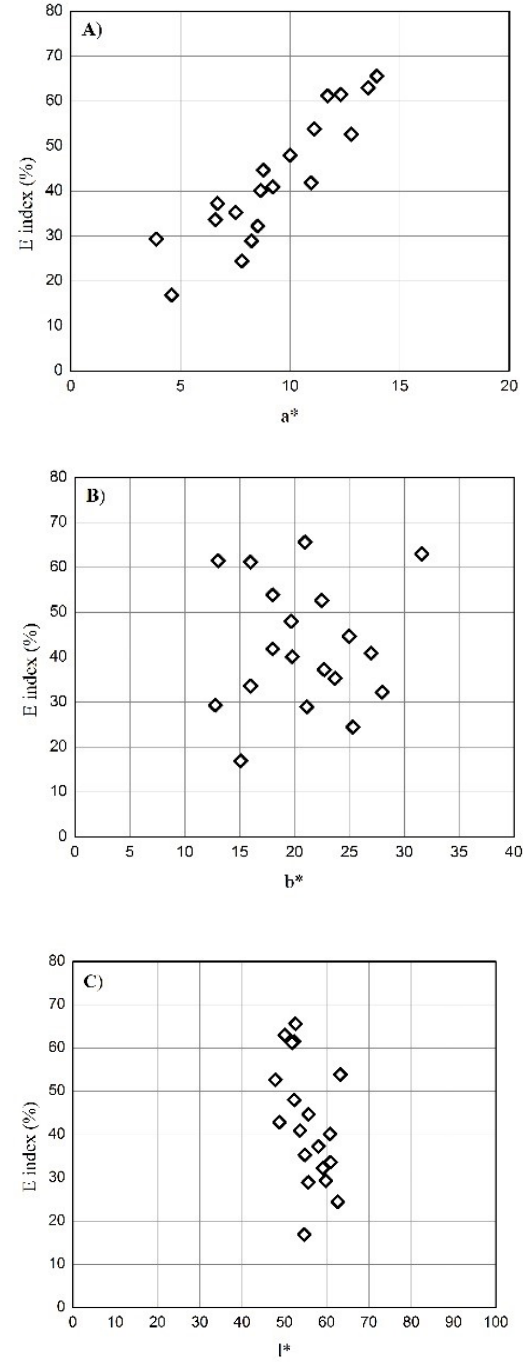

Figure 4. Comparison of Colorimetry Parameters and Erythema Index. (A) $E$ index-a*, (B) $E$ index-b*, (C) $E$ index-c*.

Table 2. Correlation and Normalization Statistics of A and E Colorimetry Factors

\begin{tabular}{|c|c|c|c|c|}
\hline \multicolumn{5}{|c|}{ Correlations } \\
\hline & & & $E$ & A \\
\hline \multirow{3}{*}{$\mathrm{E}$} & \multicolumn{2}{|c|}{ Pearson Correlation } & 1 & $0.902^{* *}$ \\
\hline & \multicolumn{2}{|l|}{ Sig. [2-tailed] } & & 0.000 \\
\hline & \multicolumn{2}{|l|}{$\mathrm{N}$} & 19 & 19 \\
\hline \multirow{3}{*}{ B } & \multicolumn{2}{|c|}{ Pearson Correlation } & $0.902^{* *}$ & 1 \\
\hline & \multicolumn{2}{|l|}{ Sig. [2-tailed] } & 0.000 & \\
\hline & \multicolumn{2}{|l|}{$\mathrm{N}$} & 19 & 19 \\
\hline \multicolumn{5}{|c|}{ One-Sample Kolmogorov-Smirnov Test } \\
\hline & & & $\mathrm{E}$ & A \\
\hline \multicolumn{3}{|c|}{$N$} & 19 & 19 \\
\hline \multirow{2}{*}{\multicolumn{2}{|c|}{ Normal parameters ${ }^{\mathrm{a}, \mathrm{b}}$}} & Mean & 42.7563 & 9.3142 \\
\hline & & SD & 14.03024 & 2.85579 \\
\hline \multirow{3}{*}{\multicolumn{2}{|c|}{ Most extreme differences }} & Absolute & 0.116 & 0.101 \\
\hline & & Positive & 0.078 & 0.101 \\
\hline & & Negative & -0.116 & -0.086 \\
\hline \multicolumn{3}{|c|}{ Test statistic } & 0.116 & 0.101 \\
\hline \multicolumn{3}{|c|}{ Asymp. sig. [2-tailed] } & $0.200^{c, d}$ & $0.200^{c, d}$ \\
\hline
\end{tabular}

**Correlation is significant at the 0.01 level [2-tailed].

${ }^{\mathrm{a}}$ Test distribution is Normal; ${ }^{\mathrm{b}}$ Calculated from data; ${ }^{\mathrm{C}}$ Lilliefors significance correction; ${ }^{d}$ This is a lower bound of the true significance. 
Table 3. Correlation and Normalization Statistics of B and E Colorimetry Factors

\begin{tabular}{|c|c|c|c|c|}
\hline \multicolumn{5}{|c|}{ Correlations } \\
\hline & & & $E$ & B \\
\hline \multirow{3}{*}{$\mathrm{E}$} & \multicolumn{2}{|c|}{ Pearson Correlation } & 1 & $0.049^{* *}$ \\
\hline & \multicolumn{2}{|l|}{ Sig. [2-tailed] } & & 0.843 \\
\hline & \multicolumn{2}{|l|}{$\mathrm{N}$} & 19 & 19 \\
\hline \multirow{3}{*}{ B } & \multicolumn{2}{|c|}{ Pearson Correlation } & $0.049^{* *}$ & 1 \\
\hline & \multicolumn{2}{|l|}{ Sig. [2-tailed] } & 0.843 & \\
\hline & \multicolumn{2}{|l|}{$\mathrm{N}$} & 19 & 19 \\
\hline \multicolumn{5}{|c|}{ One-Sample Kolmogorov-Smirnov Test } \\
\hline & & & $\mathrm{E}$ & B \\
\hline \multicolumn{3}{|c|}{$\mathrm{N}$} & 19 & 19 \\
\hline \multirow{2}{*}{\multicolumn{2}{|c|}{ Normal parameters ${ }^{a, b}$}} & Mean & 42.7563 & 20.4421 \\
\hline & & SD & 14.03024 & 5.70927 \\
\hline \multirow{3}{*}{\multicolumn{2}{|c|}{ Most extreme differences }} & Absolute & 0.116 & 0.097 \\
\hline & & Positive & 0.078 & 0.097 \\
\hline & & Negative & -0.116 & -0.080 \\
\hline \multicolumn{3}{|c|}{ Test statistic } & 0.116 & 0.097 \\
\hline \multicolumn{3}{|c|}{ Asymp. sig. [2-tailed] } & $0.200^{\mathrm{c}, \mathrm{d}}$ & $0.200^{c, d}$ \\
\hline
\end{tabular}

**Correlation is significant at the 0.01 level [2-tailed].

a Test distribution is Normal; ${ }^{\mathrm{b}}$ Calculated from data; ${ }^{\mathrm{c}}$ Lilliefors significance correction; ${ }^{\mathrm{d}}$ This is a lower bound of the true significance.

Table 4. Correlation and Normalization Statistics of I and E Colorimetry Factors

\begin{tabular}{|c|c|c|c|c|}
\hline \multicolumn{5}{|c|}{ Correlations } \\
\hline & & & $E$ & I \\
\hline \multirow{3}{*}{$\mathrm{E}$} & \multicolumn{2}{|c|}{ Pearson Correlation } & 1 & $0.503^{* *}$ \\
\hline & \multicolumn{2}{|l|}{ Sig. [2-tailed] } & & 0.028 \\
\hline & \multicolumn{2}{|l|}{$\mathrm{N}$} & 19 & 19 \\
\hline \multirow{3}{*}{ B } & \multicolumn{2}{|c|}{ Pearson Correlation } & $0.503^{* *}$ & 1 \\
\hline & \multicolumn{2}{|l|}{ Sig. [2-tailed] } & 0.028 & \\
\hline & \multicolumn{2}{|l|}{$\mathrm{N}$} & 19 & 19 \\
\hline \multicolumn{5}{|c|}{ One-Sample Kolmogorov-Smirnov Test } \\
\hline & & & E & I \\
\hline \multicolumn{3}{|c|}{$\mathrm{N}$} & 19 & 19 \\
\hline \multirow{2}{*}{\multicolumn{2}{|c|}{ Normal parameters ${ }^{\mathrm{a}, \mathrm{b}}$}} & Mean & 42.7563 & 55.5268 \\
\hline & & SD & 14.03024 & 4.62827 \\
\hline \multirow{3}{*}{\multicolumn{2}{|c|}{ Most extreme differences }} & Absolute & 0.116 & 0.116 \\
\hline & & Positive & 0.078 & 0.116 \\
\hline & & Negative & -0.116 & -0.099 \\
\hline \multicolumn{3}{|c|}{ Test statistic } & 0.116 & 0.116 \\
\hline \multicolumn{3}{|c|}{ Asymp. sig. [2-tailed] } & $0.200^{\mathrm{c,d}}$ & $0.200^{\mathrm{c}, \mathrm{d}}$ \\
\hline
\end{tabular}

**Correlation is significant at the 0.01 level [2-tailed].

a Test distribution is Normal; ${ }^{\mathrm{b}}$ Calculated from data; ${ }^{\mathrm{c}}$ Lilliefors significance correction; ${ }^{\mathrm{d}}$ This is a lower bound of the true significance.

using tissue spectroscopy and the colorimetry. Since the comparison of the absorption spectrum obtained before and after laser irradiation increased in the wavelength range of $430-610 \mathrm{~nm}$, the study was then limited to this wavelength range resulting from the absorption of hemoglobin and bilirubin pigments. An investigation of subpeaks with the correlation coefficient of 0.99 revealed the non-dependence of the resulting erythema on the bilirubin pigment, which in turn led to the selection of an effective spectral range in the wavelength range of
510-610 nm of the absorption spectrum. A statistically direct linear correlation between $\mathrm{a}^{*}$ and $\mathrm{E}$, an inverse linear correlation for $\mathrm{l}^{*}$ and $\mathrm{E}$ and no correlation for $\mathrm{b}^{*}$ and $\mathrm{E}$ showed the benefit of using Origin software CIELab colorimetry module in data analysis of spectroscopy during laser therapy.

There are some limitations in the survey. For instance, a low number of patients entered in the study, the absence of male patients as volunteers, and the not availability of all skin types in the population study. However, the result showed that the spectroscopy system could be used in side effects analysis following laser radiation. A study with the more patients of all skin types for urgent and delayed erythema assessment due to laser radiation is suggested. Spectroscopy technique can be used for analysis injuries duo to inappropriate laser radiation and also the expression of standard in the selection of skin color as the displacement for visual diagnosis medicine. It seems that in laser therapy procedures like laser hair removal, use the reflectance spectroscopy can lead more accurate and lower risk for treatment outcomes. Also, this technique suggests a beneficial tool for diagnosis and assessment of various lesions, and vascular disease and Jaundice disease in children.

\section{Conclusion}

In conclusion, optical reflectance spectroscopy is suggested as an accurate, noninvasive real-time and a complementary method for laser treatment in clinics to detect erythema as a complication and might assist clinicians to optimize the parameters based on the tissue characteristics in various candidates to achieve a better clinical outcome.

\section{Ethical Considerations}

All the participants were informed regarding the noninvasive spectroscopy tests for this research. All the participants were volunteer and their data saved based on ethical rules privately.

\section{Conflict of Interests}

The authors declare no conflict of interest.

\section{Acknowledgements}

The authors appreciate the support provided by the laser and plasma research institute, Shahid Beheshti University, and also thank Dr. Kalantari in laser clinics, for facilitating the clinical spectroscopy process.

\section{References}

1. Boer M, Duchnik E, Maleszka R, Marchlewicz M. Structural and biophysical characteristics of human skin in maintaining proper epidermal barrier function. Postepy Dermatol Alergol. 2016;33(1):1-5. doi:10.5114/ pdia.2015.48037

2. Hammes S, Karsai S, Metelmann HR, et al. Treatment errors 
resulting from use of lasers and IPL by medical laypersons: results of a nationwide survey. J Dtsch Dermatol Ges. 2013;11(2):149-156. doi:10.1111/j.1610-0387.2012.08042.x

3. Sahu RK, Mordechai S. Spectroscopic techniques in medicine: The future of diagnostics. Appl Spectrosc Rev. 2016;51(6):484-499. doi:10.1080/05704928.2016.1157809

4. LaRosa C, Chiaravalloti A, Jinna S, Berger W, Finch J. Laser treatment of medical skin disease in women. Int J Womens Dermatol. 2017;3(3):131-139. doi:10.1016/j. ijwd.2017.05.002

5. Bolognia JL, Jorizzo JL, Schaffer JV. Bolognia: Dermatology. 3rd ed. St. Louis, MO: Elsevier Saunders; 2012.

6. Argenbright LW, Forbes PD. Erythema and skin blood content. Br J Dermatol. 1982;106(5):569-574.

7. Wanner M. Laser hair removal. Dermatol Ther. 2005;18(3):209-216. doi:10.1111/j.1529-8019.2005.05020.x

8. Krishnaswamy A, Baranoski GVG. A biophysically-based spectral model of light interaction with human skin. Comput Graph Forum. 2004;23(3):331-340. doi:10.1111/ j.1467-8659.2004.00764.x

9. Tuchin VV. Light scattering study of tissues. Physics-Uspekhi. 1997;40(5):495-515. doi:10.1070/ PU1997v040n05ABEH000236

10. Nishidate I, Wiswadarma A, Hase Y, et al. Noninvasive spectral imaging of skin chromophores based on multiple regression analysis aided by Monte Carlo simulation. Opt Lett. 2011;36(16):3239-3241. doi:10.1364/ol.36.003239

11. Jaya Chandra Lekha TR, Saravana Kumar C. NIR spectroscopic algorithm development for glucose detection. International Conference on Innovations in Information,Embedded and Communication Systems
(ICIIECS); 2015. doi:10.1109/ICIIECS.2015.7192936

12. Kussw SB. Spectral imaging and analysis of human skin [thesis]. Finland: University of Eastern Finland; 2010.

13. Stamatas GN, Zmudzka BZ, Kollias N, Beer JZ. Noninvasive measurements of skin pigmentation in situ. Pigment Cell Res. 2004;17(6):618-626. doi:10.1111/j.16000749.2004.00204.x

14. Sujatha N, Anand BSS, Nivetha KB, Narayanamurthy VB, Seshadri V, Poddar R. Assessment of microcirculatory hemoglobin levels in normal and diabetic subjects using diffuse reflectance spectroscopy in the visible region-a pilot study. J Appl Spectrosc. 2015;82(3):432-437. doi:10.1007/s10812-015-0125-9

15. Nakhaeva IA, Mohammed MR, Zyuryukina OA, Sinichkin YP. The effect of an external mechanical compression on in vivo optical properties of human skin. Opt Spectrosc. 2014;117(3):506-512. doi:10.1134/s0030400x14090173

16. Koukouvinos G, Petrou P, Goustouridis D, Misiakos K, Kakabakos S, Raptis I. Development and Bioanalytical Applications of a White Light Reflectance Spectroscopy Label-Free Sensing Platform. Biosensors (Basel). 2017;7(4). doi:10.3390/bios7040046

17. Anand S, Sujatha N. Quantification of tissue oxygenation levels using diffuse reflectance spectroscopy. Tenth International Conference on Fiber Optics and Photonics; 2011. doi:10.1117/12.897811

18. Gienger J, Gross H, Neukammer J, Bar M. Determining the refractive index of human hemoglobin solutions by Kramers-Kronig relations with an improved absorption model. Appl Opt. 2016;55(31):8951-8961. doi:10.1364/ ao. 55.008951 